\title{
Der Kampf der Zensur gegen die antisemitische Propaganda in der polnisch-, tschechisch- und deutschsprachigen Presse der Donaumonarchie zur Jahrhundertwende
}

In the German press published in Opava, Silesia, at the beginning of the 20th century there was outlined the most comprehensive program of excluding Jews from the protection of otherwise commonly binding law. What prevented its popularization was the intervention of the Austrian public prosecutor as the censoring agency. The program anticipated the provisions of the later Nazi Nuremberg laws. In the Austrian monarchy, the propaganda of racial anti-Semitism that was launched by the German nationalistic press and alluded to an alleged anthropological inferiority of the Jews aimed at fostering a universal disdain toward them and promoting an acceptance of their legal exclusion. The Austrian prosecuting agency reacted sharply to these attempts and confiscated each issue of German nationalistic press products in which references to the ideology of racial anti-Semitism and appeals to the legal exclusion of the Jews were detectable.

The type of anti-Semitism that dominated in Galicia and in the Czech area was different in its nature. It had economic, cultural and religious backgrounds. It tended to evoke animosity and hostility toward Jews in order to separate them from the Christian part of society. In Galicia, attempts to resort to administrative and legal measures against the Jews and reduce them to citizens of an inferior category were rare. In the anti-Semitic texts that were censored by the Cracow or Lvov prosecuting agencies there were no open demands for the deprivation of the Jews of their public and civic rights as "a suitable method of getting rid of the pernicious influence of the Jews on Polish society". In Galicia, it was thanks to the role played in education by the conservative and catholic class, which ruled the province, that the question of abolishing equal rights for Jews did not become part of the public or political discourse.

\section{Einleitung}

Zahlreiche Beispiele von Pressezensur auf Grund antisemitischer Inhalte belegen in der Rechtsprechung in Galizien, Österreichisch Schlesien, Böhmen und Mähren, dass die k.k. Staatsanwaltschaft konsequent antijüdische Artikel eliminierte. Auch für die deutschnationale Presse in anderen Teilen der Monarchie blieb die Manifestation antisemitischer Gesinnung nicht folgenlos, wogegen etwa in Fällen, die zur nationalen Einheit mit dem deutschen http://dx.doi.org/10.1553/BRGOE2012-2s237
Kaiserreich aufriefen, einen Bismarck-Kult betrieben oder Hass den Slawen gegenüber säten, die Staatsanwaltschaft nur vereinzelt von ihren zensorischen Befugnissen Gebrauch machte. ${ }^{1}$ Die angeordneten Konfiskationen von Presseerzeugnissen wegen antisemitischer Artikel wurden fast durchgängig von den Gerichten approbiert. Selten nur fanden judenfeindliche Mei-

\footnotetext{
${ }^{1}$ HAAS, Pressegerichtliche Verfahren 221.
} 
nungen als rechtlich zulässige „Kritik ihres sozialen Wirkens“ Akzeptanz. Die Gerichte tolerierten hier insbesondere Presseaussagen über Juden als Feinde des Glaubens, der Religion und der katholischen Kirche, und unterlagen dabei den Vorurteilen von einer vorgeblichen antichristlichen Tendenz des Talmud bzw. einer besonderen Disposition dieser Glaubensgemeinschaft für den Sozialismus. ${ }^{2}$ Die Staatsanwaltschaft wandte sich in solchen Fällen an höhere Gerichtsinstanzen um Rechtshilfe und erhielt diese immer! Sie nutzte auch den Schutzschirm, den die Urteile des kaiserlichen Obersten Gerichtshofes in seiner Funktion als Revisionsgerichtshof bot, der wiederholt antisemitische Vorurteile, u.a. die Existenz des sogenannten jüdischen Ritualmordes, desavouierte. Auch

${ }^{2}$ Gegen Ende des 19. Jhs. setzte sich in vielen Kreisen die Überzeugung durch, die jüdischen Bestrebungen seien mit dem Programm der sozialistischen Bewegung ident. In der Presse, in Flugschriften und diversen Broschüren wurde oft das Stereotyp des JudenSozialisten, Feind der Kirche und des katholischen Glaubens, bemüht. Siehe: ŚLIWA, Stereotypy antyżydowskie 187. Der Antisemitismus in Galizien enthält auch kulturelle und weltanschauliche Aspekte: Das Jüdische wird als Ausdrucksform des Willens zur Vernichtung von Glauben und Kirche gesehen. Umgesetzt würde dieses durch die Gründung diverser gesellschaftlicher Bewegungen, wie Liberalismus, Freimaurerei, Sozialismus oder Feminismus. Diese antisemitische Mentalität wurde auch von Vertretern der galizischen Jurisdiktion übernommen. 1911 verweigert das LG Krakau [Kraków] die Beschlagnahme von "W Obronie Prawdy" [Verteidigung der Wahrheit] deshalb, weil es u.a. Folgendes abdruckte: „Die Juden wollen den katholischen Glauben vernichten und daher die Priester in den Schmutz ziehen. Treffen sie auf einen schlechten Priester, dann schreien sie sich heiser, was für Ausbeuter und schlechte Menschen das sind! Und wenn sie keinen finden - dann erfinden sie einen und lügen [...] ,Auf geht's gegen die Pfaffen!' Wer plärrt so? Ja, der Jude! Wer sonst, wenn nicht der Jude? Wer wiegelt sonst auf, und stachelt aus dem Verborgenen durch das Maul seiner Knechte, der Freimaurer, ,Fortschrittlichen', Sozialisten auf". Staatsarchiv Spytkowice, Landesgericht (LG) Krakau 1228 , zitiert. das Reichsgericht verurteilte bei der Verteidigung des Rechtsstaates in zahlreichen Erkenntnissen den Antisemitismus. ${ }^{3}$

\section{Antisemitische Ideologien in der polnischen und tschechischen Presse}

Bei der Bewertung eines Inhalts, der die jüdische Bevölkerungsgruppe betraf, entschied die Staatsanwaltschaft im Sinne von $\S 302$ StGB $1852^{4}$ jeweils dann auf Beschlagnahme des Druckwerks, wenn: erstens, Juden als „feindlich" - als Gefährdung der christlichen Zivilisation in wirtschaftlicher, kultureller oder rassischer Hinsicht - eingestuft wurden; zweitens ihnen bestimmte negative Eigenschaften zugeschrieben wurden; drittens ihnen unmoralisches und schädigendes Verhalten vorgeworfen wurde; und viertens in Bezug auf Juden erniedrigende und beleidigende Beschimpfungen vor-

\footnotetext{
${ }^{3}$ DZIADZIO, Monarchia konstytucyjna 116. Die Rechtsprechung der Obersten Gerichtshöfe berücksichtigte eindeutig, im Sinne einer vollständigen Delegitimation des Antisemitismus, die Vorgehensweise der kaiserlichen Verwaltungs- und Regierungsbehörden, die diesen Institutionen und Organisationen mit antisemitischen Hintergrund den Kampf ansagten und somit die verfassungsmäßige Rechtsordnung verteidigten. Vgl. die Ausführungen des Regierungsvertreters bezüglich der Auflösung des „Schulvereins für Deutsche”. in: Öffentliche Sitzung der Reichsgerichtes am 16. October 1889, AVA, II/2 Reichsgericht, Kart. 92/IV/1889.

${ }^{4} \S 302$ StGB 1852 lautete: „Wer Andere zu Feindseligkeiten wider die verschiedenen Nationalitäten (Volksstämme), Religions- oder andere Gesellschaften, einzelne Klassen oder Stände der bürgerlichen Gesellschaft oder wider gesetzlich anerkannte Körperschaften, oder überhaupt die Einwohner des Staates $\mathrm{zu}$ feindseligen Parteiungen gegen einander auffordert, aneifert oder $\mathrm{zu}$ verleiten sucht, ist, in so ferne sich diese Thätigkeit nicht als eine schwerer verpönte strafbare Handlung darstellt, eines Vergehens schuldig, und soll zu strengem Arreste von drei bis zu sechs Monaten verurtheilt werden".
} 
kamen. Nach Auffassung der Staatsanwaltschaft hatten derartige Aussagen hinsichtlich der jüdischen Bevölkerung ausschließlich das Ziel, Ablehnung und Feindschaft ihr gegenüber zu erwecken. Eine Behandlung von Tätigkeiten und des Verhaltens von Juden war nur unter Beibehaltung des „ausgeglichenen, neutralen Tons wissenschaftlicher Kritik" möglich, obwohl es der Staatsanwaltschaft in der Monarchie zur Jahrhundertwende, als die antisemitische Propaganda ihren Höhepunkt erreicht hatte, nicht immer leicht gefallen ist, die Grenze zwischen der erlaubten und verbotenen Kritik an Juden zu ziehen. $^{5}$

Die Entschlossenheit der Sicherheitsorgane in der Bekämpfung des Antisemitismus spiegelt sich nicht nur in den zahlreichen Zensurmaßnahmen der Staatsanwaltschaft zum Schutze der Juden, sondern auch in den vielen parlamentarischen Anfragen für die christlich-soziale bzw. nationalistische Presse, die immer wieder mit einer Beschlagnahme auf Grund ihrer häufigen antijüdischen Publikationen konfrontiert war.

\footnotetext{
${ }^{5}$ DzIADzIO, Antysemityzm, Die Staatsanwaltschaft trat dann nicht gegen die Verbreitung von judenkritischen Ansichten ein (die hauptsächlich in Broschüren vertrieben wurde), wenn dieser Publikation u.a. hinsichtlich der Person des Autors - einer anerkannten und respektierten Persönlichkeit aus dem Bereich, Wissenschaft, Politik oder Religion - eine ",ausgeglichene und neutrale" Qualität in der Behandlung der jüdischen Problematik zugeschrieben werden konnte. War sie jedoch der Auffassung, dass gewisse Thesen und Gedanken aus dem Inhalt beabsichtigen können, Feindschaft gegenüber den Juden hervorzurufen, dann wurden diese aus dem Text gestrichen. Ein erneuter Druck so einer Broschüre war unter Auslassung der von der Zensur beanstandeten Passagen möglich. So geschehen etwa mit „O Żydach” [Über die Juden] von Mateusz Jeż, einem bekannten Katecheten am renommierten Krakauer St. Anna Gymnasium. Hier fiel die erste Ausgabe unter die Zensur, während die nächste, mit dem Titel „Tajemnice żydowskie” [Jüdische Geheimnisse], zur Verbreitung zugelassen wurde, obwohl auch darin noch zahlreiche antijüdische Stereotypen vorkamen. Vgl.: SOBOŃ, Polacy wobec Żydów 166-167.
}

Im parlamentarischen Forum wurde die Staatsanwaltschaft angeklagt, sie wäre nicht der Wächter der verfassungsmäßigen Rede- und Pressefreiheit, sondern sei vielmehr im jüdischen Interesse als „Filiale des lokalen Rabbinats" tätig. ${ }^{6}$

Unter die Zensur fielen antijüdische Texte, unabhängig von den Motiven der jeweiligen antisemitischen Publikation. Kritik an Juden und Vorurteile konnten unterschiedlich motiviert sein: politisch, wirtschaftlich, kulturell-religiös, weltanschaulich und schließlich auch rassisch. Dies war jedoch für die Entscheidung der Staatsanwaltschaft zur Beschlagnahme nicht von größerer Bedeutung.

Das bedeutet allerdings auch nicht, dass innerhalb der gesamten Monarchie sämtliche Versionen des Antisemitismus mit der gleichen Intensität in der Presse propagiert worden wären. Die jeweilige Ausprägung unterschied sich nämlich in der polnisch-, tschechisch- und deutschsprachigen Presse. Über die Intensivierung der antijüdischen Haltung in der galizischen, böhmi-

\footnotetext{
${ }^{6}$ StenProtAH, XVI. Session. 21. 11. 1899. Die antisemitischen Vertreter beschuldigten direkt die staatlichen Strukturen, judenfreundlich zu sein, z.B.: „Die hohe Bureaukratie in Wien ist absolut in Händen der jüdischen österreichischen Union". Es wurde auch unterstellt, dass die Staatsanwaltschaft bei der Beschlagnahme der antisemitischen Presse unter jüdischem Diktat gehandelt hätte. Gegen die bei der Verfolgung antisemitischer Inhalte eifrigsten Staatsanwälte wurden beim Justizminister Anzeigen eingebracht, wie etwa gegen den Krakauer R. Doliński. Dieser bekam das Etikett „der frechste Staatsanwalt in Österreich" und wurde als "der erste und gefährlichste Anarchist in der Uniform eines k. k. Beamten" bezeichnet. Man forderte seine Amtsenthebung und sei es mittels Beförderung. Doliński wurde öffentlich z.B. wegen der Konfiskation von „Głos Narodu” [Stimme der Nation] wegen des Artikels "Krwawa żertwa” [Ein blutiges Schlachtopfer] angegriffen. Dieser wurde nämlich nicht, trotz überbordender negativer Meinungen zum Thema Juden, einschließlich der Zuschreibung des sogenannten Ritualmordes, vom Gericht der ersten Instanz zensiert. Doliński musste daher in die zweite gehen. Siehe: DzIADZIO, Antysemityzm 219 f.
} 
schen, mährischen und deutschen Presse in den 1870ern waren in erster Linie systempolitische Ursachen ausschlaggebend. Als Folge der dualistischen Ausrichtung der Monarchie und der damit verbundenen Einführung der Dezemberverfassung von 1867 kam es zu einer grundlegenden Änderung der politischen Machtverhältnisse. In Galizien und Böhmen wurden die Juden mit der Zeit aus der politischen Gemeinschaft von Polen und Tschechen ausgeschlossen. Grund hierfür war die Gleichgültigkeit gegenüber den Bestrebungen zur Änderung der zentralistischen Verfassung in Richtung einer weitgefassten sprachlichen und selbstverwalteten Autonomie. Die Deutschen ${ }^{7}$ wiederum beschuldigten die Juden, gemeinsam mit den Liberalen eine für sie günstige Verfassung $\mathrm{zu}$ forcieren, die den deutschen Interessen vollkommen entgegenstünden, da sie den nationalem Minderheiten den politischen Aufstieg sicherten.

Die Juden waren also angesichts der neuen Verfassungsrealität $\mathrm{zu}$ einer doppelten Loyalität gezwungen: gegenüber dem Haus Österreich bzw. den autonomen Kronländern und der jeweiligen Nationalität. Letztere konnte nämlich die eigene Sprache und Kultur sowie ein nationales Recht im Rahmen der eigenen Landesbehörden und Selbstverwaltungen pflegen; mit Ausnahme der Juden, die in der Monarchie ausschließlich den Status einer religiösen Minderheit genossen. Sie identifizierten sich aber ohnehin eher mit dem Kaiserreich, der deutschen Sprache und Kultur, als mit jenen Nationen, die eine politisch-rechtliche Unabhängigkeit anstrebten. Dies war also die erste Quelle für Vorurteile gegenüber dem jüdischen Bevölkerungsanteil, dessen „Fremdheit" bis dahin durch die Religion determiniert worden war, jetzt aber eine neue Dimension erfuhr.

\footnotetext{
${ }^{7}$ Hier im Sinne deutschsprachige Österreicher (Eigendefinition).
}

Durch die Distanz zu den nationalen (polnischen und tschechischen) Interessen wurden sie als politisch andersdenkende Gruppe, aber auch aus wirtschaftlichen Gründen, abgelehnt. ${ }^{8}$ Man befürchtete, dass sich die Juden in wirtschaftlicher Hinsicht eher am eigenen Gruppeninteresse, denn an den Erfordernissen des jeweiligen Landes (bzw. der Nation) orientieren würden, mit deren Bestrebungen sie sich nicht identifizierten. Das Schreckgespenst einer finanziellen und materiellen Hegemonie wurde heraufbeschworen. Mit der rechtlichen Gleichstellung ging nämlich ein intensiver wirtschaftlicher Aufstieg einher, der angesichts ihres großen Bevölkerungsanteils in Galizien, Böhmen und Mähren mit der Zeit besonders große Ausmaße annahm. U.a. angesichts des schrittweisen Rückgangs der Vermögensverhältnisse innerhalb des christlichen Bevölkerungsanteils kam es immer häufiger zu Unmutsäußerungen gegenüber Juden, einschließlich pogromartiger Ausschreitungen. ${ }^{9}$ Die Stigmatisierung von Ju-

\footnotetext{
8 In Mähren wurde der tschechisch-jüdische Konflikt, ähnlich wie in Galizien, anlässlich der direkten Wahlen zum Reichsrat politisch virulent. Der Sieg der liberalen, verfassungstreuen Seite war der Unterstützung der deutschen Beamtenschaft und Juden geschuldet, die sich damit ins Lager der Gegner der tschechischen Unabhängigkeitsbestrebungen stellten. Im Herbst 1873 erschien im "Moravská Orlice” [Der Mährische Adler] eine Artikelserie mit dem Titel „Jak jest to možné?" [Wie ist das möglich?], die von einem Grundgedanken getragen wird: Die Juden hätten sich nie mit jenen Nationen, die sie aufgenommen hatten, identifiziert. Der große Anteil am jüdischen Kapital in der Industrie um Brünn [Brno] wurde betont (laut "Adler" lebten 1843135 Juden in Brünn und 1873 bereits 4505) sowie, dass dank ihrer wirtschaftlichen Emanzipation die Stadt zu einem mächtigen Industriestandort geworden wäre. Außerdem wären die Juden hauptsächlich an der Verteidigung der eigenen Wirtschaftsinteressen interessiert. Siehe: „Moravská Orlice", Nr. 214, 18. 9. 1873, Nr. 216, 20. 10. 1873, Nr. 226, 2. 10. 1873.

${ }^{9}$ Der Regierungsapparat war, trotz seines intensiven Engagements gegen die antisemitische Propaganda, nicht im Stande, der Verbreitung von antijüdischen
} 
den als soziale Gruppe, die moralisch unter der christlichen stehe, da sie in ihrer Vorgehensweise ausschließlich von Gier getrieben, betrügerisch und hinterhältig sei, diente vor allem zur Rechtfertigung zum Kampf auf wirtschaftlicher Ebene. Obwohl die Kritik in der Presse von Anfang an die wirtschaftliche Sphäre betraf, so ist doch der Ursprung der antijüdischen Haltung in Galizien vorrangig in Zusammenhang mit dem politischen Konflikt zu suchen.

Zum politischen Bruch zwischen Polen und Juden in Galizien kam es 1873 während der direkten Wahlen zum Abgeordnetenhaus. Die Ergebnisse in Lemberg [Lwiw, Lwów] waren offensichtlich. ${ }^{10}$ Die Juden bildeten unter Einfluss der austrophilen Organisation "Szomer Israel" [,Wächter Israels"] gemeinsam mit den Ruthenen ein antipolnisches Bündnis mit Unter-

Ressentiments in der Bevölkerung entgegenzuwirken. $\mathrm{Zu}$ viele Möglichkeiten zum Austausch von Meinungen und Informationen blieben außerhalb der Kontrollmöglichkeiten, z.B. Wahlveranstaltungen (insbesondere vor den Wahlen 1897), die antijüdische Agitation des Klerus im Rahmen der Seelsorge, die Immunisierung der primitivsten Veröffentlichungen im Parlament. Der angefachte Hass gegen Juden führte in Westgalizien vom März bis Juni 1898 zum Ausbruch von gewalttätigen Tumulten (u.a. in den größeren Städten wie Neu- und Altmarkt, Limanow, aber auch in zahlreichen Dörfern und Kleinstädten der Vorkarpaten). Bauern plünderten jüdische Schenken und Geschäfte, zerstörten Eigentum und es kam zu physischen Terror gegenüber Juden. Zusätzlich angefacht wurden die damaligen Ausschreitungen durch die schwierige Situation, wegen einer vorausgegangenen Missernte. Die Behörden blieben zwar nicht untätig, schritten jedoch nicht sofort entschlossen gegen die Krawalle ein. Erst am 28. 6., als sie ein größeres Ausmaß erreicht hatten, wurde in 33 Bezirken der Ausnahmezustand verhängt. Vgl.: OLECHOWSKI, Preßrecht 508. In mehreren Ortschaften kam es zu Gewaltanwendung gegen die aufgebrachte Menge; so in Frysztak. In Folge des Gendarmerieeinsatzes verloren zwölf Teilnehmer ihr Leben. 3816 Personen, hauptsächlich Bauern und Bürger wurden angeklagt, wovon 2328 Beteiligte an den Ausschreitungen verurteilt wurden. Vgl.: SOBOŃ, Polacy wobec Żydów $240 f$.

${ }^{10}$ PIJAJ, Opozycja w wiedeńskiej Radzie Państwa $109 f$. stützung der Wiener Zentralisten. Durch diese politische Zusammenarbeit verschärfte sich das polnisch-jüdische Verhältnis. Die damaligen politischen Spannungen wurden zu einem Katalysator für die von den polnischen meinungsbildenden Eliten gehegten antijüdischen Phobien und Vorurteile, die am einfachsten auf dem Gebiet der wirtschaftlichen Rivalität avisiert werden konnten. Das politische Auftreten unter dem Schild von „Szomer Israel” scheiterte, da es den Einfluss der Polen im Reichstag nicht verhinderte. Es zeitigte jedoch negative Folgen bezüglich der polnischen öffentlichen Meinung, welche die Juden weniger als politische, denn als wirtschaftliche Gegner auffasste.

Die antipolnische Wahloffensive führte $\mathrm{zu}$ einer Gegenreaktion der Lemberger „Gazeta Narodowa" [Nationalzeitung] und des Chefredakteurs Jan Dobrzański, der die polnische Gesellschaft gegen die wirtschaftliche Dominanz mobilisierte. So veröffentlichte die Zeitung ab 1874 Fälle von Wucher und unlauteren Praktiken jüdischerseits. Dies wiederum rief eine Reaktion des "Szomer Israel” hervor, der sich mit der Forderung an den Justizminister wandte, der Hetzjagd auf die jüdische Bevölkerung einen Riegel vorzuschieben. ${ }^{11}$ Der Minister wurde aufgefordert, die "Gazeta Narodowa” unter Aufsicht der Staatsanwaltschaft Lemberg zu stellen. Die angedrohten Beschlagnahmungen sollte die Zeitung von der Verhetzung abhalten.

Die „Gazeta Narodowa” ihrerseits erkannte die ganze Angelegenheit als skandalös und verlautbare, dass sich die "galizischen Juden von der Nation losgelöst" hätten, und wurden seitens dieses meinungsbildenden Organs erstmals als polenfeindliches Element diskriminiert. Damit

\footnotetext{
11 „Gazeta Narodowa”, 27. 7. 1875, Beschlagnahmeakt auf Grund des Artikels „Glaser $i \dot{z} y d z i$ galicyjscy” [Glaser und die galizischen Juden], k. k. Oberstaatsanwaltschaft zu Lemberg, Staatsarchiv Lemberg, fond 156, opis 1, Sign. 207, siehe: BINDER, Polnisches Pressewesen $2052 \mathrm{f}$.
} 
öffnete Dobrzański hinsichtlich der antijüdischen Ressentiments in der polnischen Bevölkerung gewissermaßen die Büchse der Pandora und formierte sein Blatt zur Agitationsbühne. Hier wurden nun u.a. durch Teofil Merunowicz das polnisch-jüdische Verhältnis kritische hinterfragt und antisemitische Stereotypen gepflegt. ${ }^{12}$ Die Denkschrift der polnischen Juden goß also noch Öl ins Feuer. Auch andere Zeitungen und Zeitschriften übernahmen solidarisch die Linie der "Gazeta Narodowa”. Die sozial-wirtschaftliche Tageszeitung "Hasto" [Losung] bezeichnete die wirtschaftliche Rivalität einen „Kampf ums Dasein“ und warnte die Juden:

„So stehen also die Deutschen, Tschechen, Russen im wirtschaftlichen Kampfe gegen uns. Es wird ein verbissener Kampf, in dem ihr, die Besiegten, unserer Rat befolgen, einer ehrlichen Arbeit nachgehen, und das nationale Schicksal mit uns teilen werdet, bis ihr euch im Schweiße eures Angesichtes den Titel eines Bürgers des Landes und der Nation verdient haben werdet $^{\prime \prime} .13$

\footnotetext{
${ }^{12} \mathrm{Zu}$ antijüdischen Standpunkten siehe: DZIADZIO, Antysemityzm 215-216; SoBOŃ, Polacy wobec Żydów 148-152.

13 "Hasto", Nr. 72, 16. 9. 1874, Staatsanwaltschaft (k. k.
} Oberstaatsanwaltschaft zu Lemberg) fond 156, opis 1, Sign. 110. Auf Grund des Artikels "Żydzi” [Juden] konfiszierte die Staatsanwaltschaft 1876 die Nr. 7 des „Kurjer Lwowski” [Lemberger Kurier] u.a. dafür: „Daß heute bei vielen der Glaube schwindet, daß heute Heidentum und Gottlosigkeit auf dem Vormarsche sind, sich noch weiter verbreiten und herrschen, das ist zum größten Teile die Schuld der Juden, weil sie es sind, die in Wort und Tat, mit Zeitungen, Schriften und Büchern [...] die heidnischen Principien verbreiten”. Dabei räumte die Zeitung ein: „Es gibt Juden, die an Gott und seine Gerechtigkeit glauben [...] Wir achten sie, wir meiden sie nicht, wir leben mit ihnen. Und sei es auch der schlimmste Jude, und sollte er uns auch das größte Leide zufügen, wir verfolgen ihn nicht, wir werden nicht Böses mit Bösem vergelten [...] wir werden ihn bloß meiden. Seien wir wach-
Dieser polnisch-jüdische Wirtschaftskrieg, wie er von der "Gazeta Narodowa” angezettelt worden war, erforderte diese Art von Antisemitismus, in dem der Jude als Handelspartner, nicht vor dem Hintergrund religiöser oder rassischer Andersartigkeit, sondern über einen verdorbenen Charakter, eine räuberische und der christlichen Moral fremden Mentalität desavouiert werden sollte. Die Darstellung der Juden als Wucherer, Menschenhändler, moralische Verführer, Spekulanten und Betrüger, die in der galizischen Presse bis zum Ende der Monarchie vorherrschte, hatte vorrangig das Ziel, die christliche Bevölkerung von wie immer gearteten wirtschaftlichen oder gesellschaftlichen Verbindungen mit der jüdischen abzuhalten. ${ }^{14} \mathrm{Um}$

sam", Staatsarchiv Lemberg, fond 156, opis 1, Sign. 202 .

${ }^{14}$ Die antisemitische tschechisch-sprachige Presse erklärte ebenfalls die soziale und wirtschaftliche Trennung der christlichen und jüdischen Bevölkerung zum Ziel. Hier tauchten aber auch gewisse rassistische Merkmale auf: Die tschechische Bevölkerung wurde zur Reinhaltung des Blutes aufgefordert. Nach den antijüdischen Ausschreitungen, die im Herbst 1899 auf böhmischen Boden stattfanden, schrieb die lokale Presse, es sei kindisch, den Juden die Fenster einzuschlagen. Vielmehr sei es die "heilige Pflicht" eines jeden Tschechen, sämtliche wirtschaftliche Verbindungen zu den Juden abzubrechen. Siehe: Haus der Abgeordneten, 16. Sitzung der XVI. Session am 21. 11. 1899, 988. Die Zeitschrift "Nové Listy” [Neue Briefe] bezeichnete sich als antisemitisches, unabhängiges, politisches und nationalwirtschaftliches Tagblatt [antisemitský neodvislý politický a národnohospodáeský dennik]. 1898 veröffentlichte es seine zehn antisemitischen Gebote: „1. Du sollst das Wort Jude nicht aussprechen und nicht schreiben, wenn es nicht sein muß. 2. Handle getreu dem Motto ,jeder zu seinesgleichen" 3. Lasse die christlichen tschechischen Kaufleute und Handwerker nicht verkommen 4. Ehre und liebe dein eigenes Blut 5. Lasse nicht $\mathrm{zu}$, daß ein jüdischer Konkurrent die tschechisch christliche Industrie umbringt 6. Verhindere die Verunreinigung des tschechischen Blutes mit jüdischem 7. Bestiehle den tschechischen Kaufmann nicht dadurch, daß Du beim Juden kaufst 8. Verachte den jüdischen Schwindel 9. Verhilf nicht dem Jüdischen zur Blüte 10. Kontaktiere 
dies zu erreichen, verwendete die Presse diverse Formen der ethischen Herabwürdigung jüdischer Wirtschaftstätigkeit. Zu den populärsten Vorgehensweisen zur Geringschätzung zählten etwa die "Zehn Gebote über Juden":

„I. Du sollst mit dem Juden nicht Handel oder Schacher treiben. Der Jude frißt Dein Heim, wie so manche Villa zuvor II. Gedenke sommers wie winters, daß der Jude dein schlimmster Feind, weil er ein hiesiger ist III. Aus der jüdischen Sklaverei fliehe bei Zeiten, auf daß Du künftig nicht den Sabbath begehen musst IV. Glaube nicht des Juden Wort, da er immer lügt und Eide bricht, wenn es um's Verdienen geht V. Kauf nichts beim Juden, daß ist der aufrichtigste Rat, wer beim Juden kauft, der sich selbst bestiehlt VI. Der schlimmsten Dinge sind drei, ich zähl sie kurz auf: ein jüdischer Gutsherr, ein jüdischer Freund und der jüdische Schnaps VII Wer Schulden machen muß, meide den Juden: sein Gut und seine Ruh sind für immer dahin VIII. Nimm den Juden Dir als Pächter nicht, denn statt der Gänse wirst Du ausgenommen IX. Verkaufst du dem Juden Roß, Kalb oder Korn, fühlst Du nicht, daß du Dich selbst dabei verkaufst X. Willst Gutes Du erreichen und Böses vermeiden, gedenke stets: "Jedem die seinen “. ${ }^{15}$

Der polnische, böhmische und mährische Antisemitismus war vorgeblich der Notwendigkeit geschuldet, die wirtschaftliche nationale Souveränität zu erkämpfen. Er sagte sich von der nicht selten angewandten physischen Gewalt los und betrachtet den Boykott als bestes Mittel. Die galizische und tschechisch-sprachige Presse forderte nicht den Ausschluss der Juden von der verfassungsmäßigen Gleichbehandlung aller Bürger vor dem Gesetz, wie es für den deutschen, rassischen Antisemitismus bezeichnend war. Die galizische Variante war vor allem ein

niemals deinen jüdischen Feind.“ Staatsarchiv Prag [Praha],LG Prag, Kart. 1044, zitiert.

${ }^{15} \mathrm{Im}$ polnischen Original gereimt "Nowy Dzwonek", 15. 2. 1914, Nr. 4, LG Krakau, Sign. Pr III 13/14.
Mäntelchen für die wirtschaftliche Auseinandersetzung. Die Zeitschrift „Wawel”, das Organ des Polnischen Nationalverbandes [Polski Zwiazek Narodowy], beklagte 1911 das Fehlen einer Partei, die „auf ökonomischer Basis unter dem Banner des Antisemitismus" auftrete. Die eigene Linie definierte sie, folgendermaßen:

„Unser Antisemitismus reguliert nicht unser Handeln und ist nicht die Basis unserer Ansichten, sondern Ausdruck des Selbsterhaltungstriebes und eine Konsequenz aus dem Verständnis des nationalen Interesses. Da wir sehen, daß die Juden ihre soziale Eigenart strikte beibehalten und in unserem Lande nicht nur leben [...] sondern auch gezielt auf unsere Vertreibung aus unseren Ämtern hinarbeiten, und dabei bei den Mitteln nicht wählerisch sind, so ist die Aufnahme eines energischen Kampfes gegen sie sowohl ein Gebot des nationalen, polnischen Interesse, als auch des einfachen Gerechtigkeitsempfindens ". .16

Einen ähnlichen Tonfall schlugen die tschechischsprachigen Zeitungen mit einem christlich-sozialen Profil an. Sie waren der Meinung, der Antisemitismus wäre weder religiös, noch rassisch bedingt, sondern hätte einzig politische und wirtschaftliche Ursprünge. Sie vertraten auch die Ansicht, dass Juden in Übereinstimmung mit dem fortschrittlichen Grundsatz der Gleichheit aller vor dem Gesetz, im gleichen Maße Gebrauch von ihren Bürgerrechten machen sollten, wie alle anderen gesellschaftlichen Gruppen. ${ }^{17}$ Dagegen fand die Rassentheorie von

\footnotetext{
16 „Wawel" Nr. 13, 15. 11. 1911,LG Krakau 1231. Als geeignetes Mittel im Kampf gegen die Juden forderte die Zeitschrift: 1) Den Boykott sämtlicher jüdischer Geschäfte, Lagerhäuser und Fabriken, 2) Den Boykott jüdischer Zinshäuser, 3) den Einsatz der Tagespresse zur Unterstützung des Boykotts sowie die Bekanntmachung der Kundennamen von jüdischen Verkäufern, 4) jüdische Firmen als solche in einer Liste auszuweisen.

17 "Nové Listy", Nr. 41, 7.10. 1899. In einem Artikel „Židovska otázka - otázka sociálni" [Die jüdische Frage -
} 
der anthropologischen Andersartigkeit und der Minderwertigkeit der jüdischen Nation, wie sie von der deutschnationalistischen Presse propagiert wurde, kaum Anhänger unter den polnischen und tschechischen Antisemiten, obwohl die antijüdisch chauvinistische Presse auch Schlesien, Böhmen und Mähren in einem größeren Umfang erreichte. ${ }^{18}$ In Galizien vertrat Bohdan Czajkowski publizistisch den rassistischen Standpunkt in der Judenfrage. Er war Mitarbeiter der Geistlichen Stanisław Stojałowski und Jan Badeni. Mit ihnen kam es jedoch zum Zerwürfnis, da er zur Rettung der christlichen Gemeinschaft vor der Überflutung durch die "fremde Rasse“ aufrief. Seine rassistischen Thesen propagierte er in der Zeitschrift "Naród" [Nation], die folglich häufig beschlagnahmt wurde. Wegen antisemitischer Propaganda kam es zu 16 Gerichtsprozessen gegen ihn. Er verbüßte auch mehrfach Haftstrafen, wegen tätli-

eine soziale Frage] hieß es: „Es steht außer Frage, daß jeder dumm und ungerecht handeln würde, der eine Verabschiedung von Sondergesetzen für Juden forderte. Wir gehen vom Princip der vollständigen bürgerlichen Gleichheit aus - das ist ein Princip, von dem jeder fortschrittliche Mensch ausgehen muß - wir müssen den Juden das gleiche Maß an bürgerlichen Freiheiten zugestehen, wie allen anderen" Die Zeitung propagierte also einen politisch motivierten Antisemitismus und berief sich auf einen wirtschaftlichen Widerstand, der die Konsumenten vor - dem hauptsächlich jüdischem - Wucher schützen sollte. Staatsarchiv Prag, LG Prag, Kart. 1044.

${ }_{18}$ Als Beispiel seien die zahlreichen Beschlagnahmen antisemitischer Schriften, die der Leipziger Verlag „Hammer” 1912 in Österreichisch Schlesien vertrieb, erwähnt. In einem war zu lesen: „Sind sie von Eurem Blut, Ehre Rasse?? Nein sie sind allesamt von einem fremden Stamme, der mit unserem deutsche Volke nichts zu tun hat - und nicht zu tun haben will [...] Er muss dieses störenden Element, dieser sociale Krankheits-Bazillus beseitigt sein, der alle Harmonie im Volkskörper verhindert [...] Wer einem Juden oder Juden-Genossen seine Stimme gibt, der begeht einen Verrat am deutschen Volke, am eigenen Blute und Leibe". Staatsarchiv Teschen [Cieszyn], BG Teschen, 14/43, Sign. 422-427. cher Angriffe gegen - seiner angeblichen Diktion nach - "räudige" Juden. Seine Ansichten stießen allerdings auf kein größeres Echo, mit Ausnahme des kleinen Milieus von Lemberger Bürgern und Kaufleuten. ${ }^{19}$

\section{Der Anstieg antisemitischer Haltungen zur Jahrhundertwende}

Gegen Ende des 19. Jahrhunderts gab es eine richtiggehende "Schwemme" antisemitischer Schriften, sowohl in der galizischen, als auch in der tschechisch- sowie deutschsprachigen Presse. Wie bereits erwähnt war hierfür ein Prozess und dessen weitreichendes Echo mit Ausschlag gebend. Dieser fand 1899 im mährischen Polna statt und betraf einen angeblichen Ritualmord („Hilsneriade“). Er rief in der gesamten Monarchie eine antijüdische Hysterie hervor, die der französischen Dreyfuss-Affäre kaum nachstand. Die Jahrhundertwende war also für die kaiserliche Staatsanwaltschaft eine Zeit intensiver Beschlagnahmungen, mit denen man die antisemitische Propaganda einzudämmen versuchte, deren Ausmaße erstmals derart gewaltig waren. In diese Zeit fallen auch die ungeschlachten Angriffe gegen die Staatsanwaltschaft auf parlamentarischer Ebene, die an Vulgarität und Primitivität kaum zu übertreffen waren. ${ }^{20}$

${ }^{19}$ Staatsarchiv Lemberg, LG Lemberg, fond 152, opis 2a, Sign. 1081. Im Juli 1899 konfiszierte die Staatsanwaltschaft eine von Lemberger Bürgern (J. Bojarski, S. Kolankowski, J. Białek, F. Wójcicki) unterzeichnete Aufforderung, die zu einer Sammelaktion aufrief, um die Geldstrafe, die gegen B. Czajkowski verhängt worden war, zu begleichen. Dieser wurde von seinen Verteidigern als ",edler Fanatiker“ bezeichnet.

${ }^{20}$ StenProtAH, XVII. Session, 28. 3. 1901， 2078. Die Deutschnationalen mit Schönerer unternahmen zahlreiche Vorstöße, um Artikel der antisemitischen Presse zu immunisieren, in denen die Juden als "Seuche“ oder „Vampir, der das Christenblute saugt" bezeichnet worden waren. Als Beispiel mag folgendes Zitat 
Die mit dem Hilsner-Prozess verbundene Pressekampagne blieb in Bezug auf die öffentliche Meinung nicht folgenlos. Im Herbst 1899 kam es in Mähren zu gegen Juden gerichtete Ausschreitungen. Juden hätten Privilegien von staatlicher Seite zu Ungunsten der Tschechen genützt, denen die Regierung in diesen Tagen endgültig die Gleichberechtigung absprach, indem sie die zuvor erlassene Sprachenverordnung aufhob. Die Krawalle waren also auch dezidiert regierungsfeindlich. ${ }^{21}$ Die behördlichen Interventionen zogen Todesopfer nach sich, was sich nicht nur in einem breiten Medienecho niederschlug, sondern auch unzählige Abgeordnetenanfragen nach sich zog, die wiederum in erster Linie von als antisemitisch bekannten Vertretern stammten. Auf Basis der blutigen Tumulte in Mähren (u.a. in Prerau [Přerov] oder Holleschau) kam es im Wiener Parlament zu ersten Forderungen,

aus der „Nationalen Zeitung” dienen: „, Wir werden den Kampf gegen die überhandnehmende Verjudung nicht nur im Ärztestande, sondern auch in anderen akademischen Berufen führen und hoffen, daß es uns schließlich gelingen wird, die jüdischen Pest-Beulen auszurotten, was nur zum Heile unseres Volkes gereichen wird".

21 "Česká Samostatnost" [Tschechische Unabhängigkeit] Nr. 29, 1. 12. 1899. Staatsarchiv Brünn, LG Brünn, Kart. 587. Die Zeitung schrieb über die Strafsachen gegen die Teilnehmer an den Vorfällen in Boskovice. Sie gab an, dass ihr Mitarbeiter als Aufrührer der antisemitischen und regierungsfeindlichen Krawalle angeklagt sei. Ihm wurde vorgeworfen folgende Ausrufe getätigt zu haben: „židi̊m šibenice” [Juden an den Galgen], "pojd'te na židy" [Auf gegen die Juden!], „Hilsner", "hanba vládé" [Schandregierung]. Impulsgeber für die antijüdischen Ausschreitungen waren manchmal Repressalien der Behörden gegen die Herausgeber antisemitischer Schriften. Sie brachten die Bevölkerung gegen die Juden mittels Berichterstattung über den Hilsner-Prozess auf, die in der Regel von der Beschreibung historischer Ritualmorde begleitet wurden. Die lokale Bevölkerung betrachtete dies als Behördenwillkür und die Verfasser als Opfer einer jüdischen Hetzkampagne; in diesem Sinne auch die Ereignisse in Holleschau [Holešov], wo fünf Angreifer auf jüdisches Eigentum unter den Kugeln der Gendarmerie ihr Leben ließen. die bürgerlichen und öffentlichen Rechte von Juden einzuschränken. ${ }^{22}$

Im Abgeordnetenhaus erhoben sich nur wenige Stimmen, die gegen die Anschuldigung der Ritualmordpraxis per se protestierten. Ausnahmslos die gesamte antisemitische Presse der Monarchie engagierte sich zum Beweis des Gegenteils. Dazu diente eine Liste konkreter Fallbeispiele der Ermordung von Christen durch Juden für rituelle Zwecke, die angeblich in Europa vom Mittelalter bis ins 19. Jh. stattgefunden haben sollen. ${ }^{23} \mathrm{Zu}$ jenen Politikern, die sich sei-

22 StenPRotAH, XVI. Session, 27. 11. 1899, 1283. Während dieser Sitzung traten einige Abgeordnete (darunter Wohlmeyer und Schlessinger) mit der Regierungsanfrage auf, wann die jüdische Frage in Österreich einer „endlichen Regelung” zugeführt werde bzw. Gesetze eingeführt würden, welche die christliche Bevölkerung vor den Juden schützten. Die Antragsteller forderten von der Regierung eine Beschränkung der wirtschaftlichen Berechtigungen für Juden. Des Weiteren verlangten sie eine staatliche Kontrolle in dieser Hinsicht, die Ausweisung von Juden ohne Staatsbürgerschaft, ein Immigrationsverbot für Juden, den Ausschluss von Juden vom Richteramt und anderen Positionen öffentlichen Vertrauens sowie von Professorenstellen, das Verbot für jüdische Lehrer, christliche Kinder $\mathrm{zu}$ unterrichten bzw. für jüdische Ärzte in öffentlichen Krankenhäuser, christliche Patienten zu behandeln.

23 "Lidový List" [Volksblatt], Nr. 9, 1899, Staatsarchiv Prag, LG Prag, Kart. 1043. Ein Beitrag mit dem Titel "22 ritelnich vrażd" [22 Ritualmorde] wird mit dem Satz: "das sind historische Tatsachen”, abgeschlossen. Die antisemitisch eingestellte Presse führte solche angeblichen Fakten an, um sich Prof. T. Masaryk entgegenzustellen. Der spätere Präsident der Tschechoslowakei kritisierte mehrfach die Vorgehensweise und Einstellung des Gerichtes in Kuttenberg [Kutna Hora]. Er bekämpfte das Vorurteil des jüdischen Ritualmordes und forderte diesbezüglich eine Revision des Prozesses. Er glaubte, das Opfer sei in einem geschlossenen Raum ermordet worden und erst später zum Auffindungsort verbracht worden, um den Verdacht auf die Juden zu lenken. Masaryk wurde beschuldigt, unrechtmäßig den Prozessverlauf noch vor der Urteilsverkündigung publik zu machen. Trotzdem wurden seitens der Staatsanwaltschaft Behauptungen der Presse, Masaryk sei Philosemit 
nerzeit an parlamentarischen Anfragen gegen die Verbreitung dieses antisemitischen Vorurteils engagierten, gehörten vorrangig die Konservativen aus Galizien. Die parlamentarische Intervention polnischerseits wurden u.a. durch die Information hervorgerufen, dass sich der Staatsanwalt in seinem Plädoyer in Kuttenberg auf ,bekannte Motive” berief, und somit die antijüdischen Klischeevorstellungen rechtfertigte. Es wurden daher entschlossene Maßnahmen seitens des Justizministers gegen die Herausgeber jener Zeitungen und Zeitschriften eingefordert, welche die Annahme verbreiteten, Juden würden für rituelle Handlungen Christenblut benötigen. In der Begründung der Anfrage berief man sich im großen Ausmaß auf die Gesetzgebung der polnischen Könige, die jedem die Todesstrafe androhten, die Juden eines Ritualmordes bezichtigten. ${ }^{24}$

und handle im Auftrag des jüdischen Kapitals, zensiert. Die Zeitung "České Zájmy” [Tschechische Interessen], Nr. 392, 20.12.1899, Staatsarchiv Prag, LG Kart. 1044, schrieb: "Zde Masaryk - tam Zola!" [Hier Masaryk - dort Zola!]. Damit beschuldigte sie Masaryk, er würde, wie Zola Dreyfuss in Frankreich, Hilsner in Östereich verteidigen; selbstverständlich als bezahlter Büttel der Juden: „Der ehrliche, treue Tscheche sammelte bereits gegen Ende November 100.000 Kronen, um eine Tageszeitung für die Bekämpfung des Klerikalismus und Antisemitismus und zum Schutze des jüdischen Schurken, des notorischen Feindes, zu gründen". Die České Zájmy wurden mit Konfiskationen eingedeckt. In der Mehrzahl verlautbarten die tschechischen Zeitungen, dass der Mord in Polna von gerichtlicher Seite als Ritualmord eingestuft wurde. Vgl. z.B. "Česka Straž” [Die tschechische Wacht], Nr. 38, 20. 10. 1899, "Katolické Listy" [Katholische Briefe], Nr. 266, 26. 10. 1899, „Nove Listy” [Neue Briefe], Nr. 42, 14. 10. 1899, Staatsarchiv Prag, LG Prag, Kart. 1041.

${ }^{24}$ StenProtAH, XVI. Session, 18. 10. 1899, 35. Die Interpellation wurde u.a. von Abrahamowicz, Piętak, Dzieduszycki, Biliński, Milewski, Roszkowski, Jaworski, Sokołowski, Górski und Bogdanowicz unterzeichnet. Der einzig deutsche Unterstützer war Dr. Straucher, der seinerseits 1907 eine parlamentarische Anfrage an den Justizminister bezüglich einer Neuaufnahme des Hilsner-Prozesses einbrachte. Der
Der Auftritt der polnischen Abgeordneten stieß auf heftigen Widerstand seitens der Deutschnationalen: Sie sollten sich, anstatt die Juden aus rein humanitären Gründen zu verteidigen, besser auf die Verteidigung der christlichen Bevölkerung vor der jüdischen Ausbeutung konzentrieren. Die Deutschnationalen verteidigten also die galizische Bevölkerung, die ja nicht auf die eigenen Vertreter vertrauen könne, indem sie an die Regierung die Frage richteten, was diese zum Schutz der galizischen Bauern vor den Juden zu tun gedenke. Die deutschen Antisemiten führten Statistiken an, welche das Ausmaß der Ausbeutung galizischer Bauern durch die Juden belegen sollten. Die polnischen Grundbesitzer wurden bezichtigt, einen Wirtschaftspakt mit den Juden eingegangen $\mathrm{zu}$ sein, um die Landbevölkerung in ökonomischer und politischer Hinsicht in Abhängigkeit zu halten. ${ }^{25}$

\section{Antisemitische Propaganda in der deutschsprachigen Presse}

Dass der Einspruch polnischer Politiker gegenüber der Beschuldigung sogenannter Ritualmorde seitens der Deutschnationalen angeprangert wurde, zeigt, wie der galizische Antisemitismus durch die humanitäre Einstellung der Eliten gemildert wurde; im Gegensatz zum deutschen Antisemitismus, der hauptsächlich von den politischen Führern der nationalen Bewegung verbreitet wurde. Es waren nämlich andere Prämissen, die über den Ausschluss von Juden aus der Zugehörigkeit zur deutschen kulturellen und politischen Sphäre entschieden, an deren Schaffung sie ja mitwirken wollten. Der

geistig zurückgebliebene Hilsner verbüßte eine Freiheitsstrafe und wurde vom Antragsteller als unschuldiges Opfer einer antisemitischen Pressekampagne bzw. als Paradebeispiel eines "Justizmordes“ angesehen.

${ }^{25}$ StenProtAH, XVI. Session, 21. 11. 1899, 990. 
Antisemitismus der Deutschnationalen konnte sich daher nicht nur mit politischen, wirtschaftlichen oder kulturell-weltanschaulichen Kriterien auf eine Definition des Jüdischen als etwas „Fremdes" (das dem Deutschtum feindlich entgegenstehen würde) beschränken. Er musste sich gezwungenermaßen auch auf ethnische bzw. rassische Voraussetzungen berufen, denn nur so war ihnen das Recht auf Akkulturation mit der deutschen Nation [!] zu nehmen und nur derart konnten sie vom Einfluss auf die Ausformung der deutschen Zivilisation, wie sie das Deutsche Kaiserreich und die österreichischen Anhänger verstanden, abgeschnitten werden. „Durch [rassische] Reinheit zur [deutschen] Einheit!" - lautete der Untertitel einer österreichischen deutschnationalen und antisemitischen Postille. ${ }^{26}$

Die nationalistische Presse übertraf sich in Schmierereien bezüglich der rassischen Andersartigkeit von Juden und deren Minderwertigkeit gegenüber der arischen Rasse. Auf jegliche erdenkliche Weise wurden Juden menschliche Merkmale abgesprochen. Diese Dehumanisierung der jüdischen Bevölkerungsgruppe sollte die soziale Akzeptanz dafür schaffen, sie künftig außerhalb des Rechtsschutzes zu stellen. Die deutschnationale Presse Österreichs propagierte somit erstmals in der westeuropäischen Geschichte ein Gesetzesprojekt, das die bürgerlichen und öffentlichen Rechte einer sozialen Gruppe, einzig aus rassischen Gründen, einzuschränken gedachte. Daher war sie die unrühmliche Vorläuferin der Nürnberger Rassengesetze. $\mathrm{Zu}$ diesen Zeitschriften, die zur Jahrhundertwende ein Programm zur Verweigerung von Bürger- und Privatrechten für Juden vertraten,

\footnotetext{
26 „Volks=Frühling Jungdeutschland", Nr. 17, Oktober 1899. Diese Ausgabe wurde wegen eines Artikels beschlagnahmt, in dem die Meinung vertreten wurde, die mindere jüdische Moral, die auch in der christlichen Religion enthalten sei, passe nicht zur „besseren deutschen Seele“.
}

gehörte die „Deutsche Wehr”. Sie erschien in Troppau [Opava] und vertrat in Schlesien eine antisemitische Propaganda im Sinne von Georg von Schönerers Erweiterung des Linzer Programms. ${ }^{27}$

Der hier publizierte Vorschlag einer rechtlichen Regelung, die von einem Ausschluss der Juden vom geistigen, nationalen und öffentlichen Leben ausging, wurde vor allem rassisch begrün$\operatorname{det}^{28}$. Der Autor war überzeugt, dass die Juden einer minderen - von Natur aus abartigen und unmoralischen - Rasse angehören, welche die Kultur und Sitten besserer moderner Nationen beeinträchtigten, weshalb sie mit diesen niemals rechtlich gleichgestellt werden könnten. Für die Deutschnationalen in Cisleithanien war die jüdische Frage weder eine religiöse, noch eine soziale oder wirtschaftliche, sondern rein rassisch: „Die Judenfrage ist eben in erster Linie eine Charakterfrage". Als Charakteristikum des Jüdischen galt also ausschließlich die Rasse, da in einer sich laisierenden Welt die Religion nicht mehr als Basis für die Identifikation eines Juden ausreichte (,Als Kennzeichen des Judenthums ist nur die Rasse zu betrachten"). Allein das Rassekriterium würde die Beschränkung der Bürgerrechte der gesamten jüdischen Bevölkerungsgruppe ermöglichen. 1901 forderte daher die „Deutsche Wehr” die Einführung rechtlicher Mittel, um Folgendes zu erreichen:

a) Ausschließung der Juden aus öffentlichen Ämter, aus dem Rechtsanwalts- und Ärztestan-

\footnotetext{
27 „Deutsche Wehr", Nr. 71, 4.9. 1901, Staatsarchiv Troppau, LG Troppau Kart. 697, zitiert. Siehe auch: HÖBELT, Deutsche Presselandschaft 1862f.

${ }^{28}$ Der Autor war ein gewisser Matthäus Joksch, der mit der Deutschen Volkspartei in Verbindung stand. Seine Ansichten wurden von zahlreichen Vertretern der nationalen Richtung in Österreich geteilt, u.a. von Schönerer, oder Wolf. Vgl. die Interpellation der Deutschnationalen gegen die Beschlagnahme des Joksch-Artikels, in: StenProtAH, XVI. Session, 8.6. 1900, 3699.
} 
de und aus den Lehrerkollegien deutscher Schulen, sowie aus der deutschen Presse; ${ }^{29}$

b) Ausschließung der Juden aus Armee. Diese Ausschließungen gelten auch für Deutsche, welche Jüdinnen geheiratet haben und erstreckt sich weiter auf dessen Nachkommen bis ins dritte Glied; 30

c) Verbot der Judeneinwanderung;

d) Verbot, Grund und Boden zu besitzen;

e) Verbot, weibliches Personal arischen Stammes zu halten;

f) Unterricht in besonderen Schulen;

g) Verbot der Führung arischen Namen und Firmen;

h) Einrichtung einer Judenstatistik;

i) Ausschließung der Juden von der direckten und indireckten Betheiligung an öffentlichen Lieferungen, Verpachtungen und Submissionen. ${ }^{31}$

\section{Schluss}

Zu Beginn des 20. Jahrhunderts entwickelte sich also in der deutschnationalen Presse der Monarchie das weitreichendste Programm zur Entrechtung von Juden. Die rassistische antisemitische Propaganda mit dem Tenor, die Juden seien bereits allein auf Grund ihrer Abstammung schlechter als Arier, zielte auf eine allgemeine Verunglimpfung ab und bereitete deren rechtlichen Ausschluss vor. Der wirtschaftliche oder kulturell-religiös geprägte Antisemitismus, wie

${ }^{29} 1$. Verordnung zum Reichsbürgergesetz vom 14. 11. 1935, § 4 Z. 1. „Ein Jude kann nicht Reichsbürger sein. Ihm steht ein Stimmrecht in politischen Angelegenheiten nicht $\mathrm{zu}$; er kann ein öffentliches Amt nicht bekleiden.", dRGBl. I, 125.

${ }^{30}$ Ebd.: § 5 Z. 1. "Jude ist, wer von mindestens drei der Rassen nach volljüdischen Großeltern abstammt." ${ }^{31}$ Vgl. die NS-Verordnung vom 12.11. 1938: "Ausschaltung der Juden aus dem deutschen Wirtschaftsleben" dRGBl. I, 1580. er in Galizien oder Böhmen dominierte, zielte dagegen auf eine feindschaftliche Atmosphäre den Juden gegenüber $a b$, um diese vom Rest der christlichen Gesellschaft abzusondern. Forderungen nach administrativen und rechtlichen Mittel gegen Juden, um diese zu Bürgern zweiter Klasse zu degradieren, waren in Galizien äußerst selten. In den von der Krakauer oder Lemberger Staatsanwaltschaft beschlagnahmten Texten mit antisemitischen Inhalt finden sich keine eindeutigen diesbezüglichen Forderungen, Juden bürgerliche oder öffentliche Rechte abzuerkennen, um mit diesem Mittel deren „verhängnisvollen Einfluss“ auf die polnische Gesellschaft einzudämmen. Zweifellos hatten die lokalen konservativen und katholischen Regierungskreise hier einen erzieherischen Einfluss. Sie verhinderten, dass die Frage der Aufhebung der Gleichberechtigung von Juden Teil der öffentlichen oder politischen Debatte wurde.

\section{Korrespondenz:}

Dr. hab. Andrzej Dziadzio Uniwersytet Jagielloński

Katedra Powszechnej Historii Państwa I Prawa

ul. Gołębia 9, 31-007, Kraków, Polska

andrzej.dziadzio@uj.edu.pl 


\section{Abkürzungen:}

StenProtAH Stenographische Protokolle des Abgeordnetenhauses

\section{Literatur:}

Harald BINDER, Das polnische Pressewesen, in: Helmut Rumpler, Peter Urbanitsch (Hgg.), Die Habsburgermonarchie 1848-1918, Bd. VIII: Politische Öffentlichkeit und Zivilgesellschaft, 2.Teilbd.: Die Presse als Faktor der politischen Mobilisierung (Wien 2006) 2037-2090.

Andrzej DzIADZIO, Monarchia konstytucyjna w Austrii 1867-1914. Władza Obywatel Prawo, (Kraków 2001) 116.

Andrzej DZIADZIO, Antysemityzm jako powód konfiskat prasowych. Orzecznictwo sądów krakowskich (XIX/XX w.), in: Cuius Regio Eius Religio? Wydawnictwo KUL, Bd. II (Lublin 2007) 75110.

Hanns HAAS, Pressegerichtliche Verfahren in Salzburg 1862-1918, in: Erika Weinzierl u.a. (Hgg.),
Justiz und Zeitgeschichte. Symposiumsbeiträge 1976-1993, Bd. 2 (Wien 1995) 207-226.

Lothar HÖBELT, Die deutsche Presselanschaft, in: Helmut Rumpler, Peter Urbanitsch (Hgg.), Die Habsburgermonarchie 1848-1918, Bd. VIII: Politische Őffentlichkeit und Zivilgesellschaft, 2.Teilbd.: Die Presse als Faktor der politischen Mobilisierung (Wien 2006) 1819-1894.

Thomas OlechOWSKI, Die Entwicklung des Preßrechts in Österreich bis 1918 (Wien 2004).

Stanisław PIJAJ, Opozycja w wiedeńskiej Radzie Państwa w latach siedemdziesiątych XIX w. (skład-organizacja-funkcjonowanie), (Kraków 2011).

Michał ŚLIWA, Stereotypy antyżydowskie w konserwatywno-katolickiej krytyce socjalizmu, in: Myśl i polityka. Księga pamiątkowa dedykowana profesorowi Jackowi Marii Majchrowskiemu, in: Bogdan SzlachTA (Hg.), „O myśli politycznej” (Kraków 2011), 187.

M. SoBoŃ, Polacy wobec Żydów w Galicji doby autonomicznej w latach 1868-1914 (Kraków 2011). 